TTP96-43

hep-ph/9609502

September 1996

\title{
CP Violation in Semileptonic $\tau$ Decays with Unpolarized Beams
}

\author{
J.H. Kühn and E. Mirkes \\ Institut für Theoretische Teilchenphysik, Universität Karlsruhe, \\ D-76128 Karlsruhe, Germany
}

\begin{abstract}
$\mathrm{CP}$ violating signals in semileptonic $\tau$ decays are studied. These can be observed in decays of unpolarized single $\tau$ 's even if their rest frame cannot be reconstructed. No beam polarization is required. The importance of the two meson channel, in particular the $K \pi$ final state is emphasized.
\end{abstract}




\section{INTRODUCTION}

$\mathrm{CP}$ violation has been experimentally observed only in the $K$ meson system. The effect can be explained by a nontrivial complex phase in the CKM flavour mixing matrix [1]. However, the fundamental origin of this $\mathrm{CP}$ violation is still unknown. In particular the $\mathrm{CP}$ properties of the third fermion family are largely unexplored. Production and decay of $\tau$ leptons might offer a particularly clean laboratory to study these effects. CP-odd correlations of the $\tau^{-}$and $\tau^{+}$decay products, which originate from an electric dipole moment in the $\tau$ pair production, are discussed in [2,[3]. In this paper, we investigate the effects of possible non-Kobayashi-Maskawa-type of $\mathrm{CP}$ violation, i.e. CP violation effects beyond the Standard Model (SM) on semileptonic $\tau$ decays. Such effects could originate for example from multi Higgs boson models [4].

$\mathrm{CP}$ violation effects in the $\tau \rightarrow 2 \pi \nu$ decay mode from this scalar sector have recently been discussed in terms of "stage-two spin correlation functions" in [5] and in the case of polarized electron-positron beams at $\tau$ charm factories in [6]. CP violation in the three pion channel has been also discussed in [7] and in the $K \pi \pi$ and $K K \pi$ channels in [8], where the latter analysis is based on the " $T$-odd" correlations as derived in [9] and the vector meson dominance parameterizations in [10].

In the present paper we show that the structure function formalism in [9] allows for a systematic analysis of possible CP violation effects in the two and three meson cases. Special emphasis is put on the $\Delta S=1$ transition $\tau \rightarrow K \pi \nu_{\tau}$ where possible CP violating signals from multi Higgs boson models would be signalled by a nonvanishing difference between the structure functions $W_{S F}\left[\tau^{-} \rightarrow(K \pi)^{-} \nu_{\tau}\right]$ and $W_{S F}\left[\tau^{+} \rightarrow(K \pi)^{+} \nu_{\tau}\right]$. Such a measurement is possible for unpolarized single $\tau$ 's without reconstruction of the $\tau$ rest frame and without polarized incident $e^{+} e^{-}$beams. It is shown that this difference is proportional to $\operatorname{IM}($ hadronic phases $) \times \operatorname{IM}(\mathrm{CP}$-violating phases $)$, where the hadronic phases arise from the interference of complex Breit-Wigner propagators, whereas the $\mathrm{CP}$ violating phases could arise from an exotic charged Higgs boson. An additional independent test of $\mathrm{CP}$ violation in the two 
meson case would require the knowledge of the full kinematics and $\tau$ polarization.

The paper is organized as follows: CP violating terms in the Hamiltonian for $\tau$ decays are discussed in section II. It is shown that CP violating signals induced through the exchange of an exotic intermediate vector boson can only arise, if both vector and axial vector hadronic currents contribute to the same final state, i.e. for final state which are not eigenstates of $G$ parity and involve three or more mesons. The kinematics and the relevant form factors and structure functions for tests of CP violation in the two meson case are presented in section III. $\mathrm{CP}$ violation effects in three meson final states are briefly discussed in section IV. Finally, it is shown in appendix A that the reconstruction of the full kinematics is possible for the case, where one $\tau$ decays into one charged hadron and the second $\tau$ decays into a $\rho$ or $K^{*}$ with the subsequent decay into a neutral and a charged meson.

\section{CP VIOLATION IN $\tau$ DECAYS}

The Hamiltonian responsible for $\tau$ decays is decomposed into the conventional term of the SM, denoted by $H_{S M}$, a CP violating term of similar structure, induced e.g. by the exchange of a vector boson, $H_{C P}^{(1)}$ and a $\mathrm{CP}$ violating term induced by scalar or pseudo scalar exchange $H_{C P}^{(0)}$ :

$$
\begin{aligned}
& H_{S M}=\cos \theta_{c} \frac{G}{\sqrt{2}}\left[\bar{\nu} \gamma_{\alpha}\left(g_{V}-g_{A} \gamma_{5}\right) \tau\right] \quad\left[\bar{d} \gamma^{\alpha}\left(1-\gamma_{5}\right) u\right]+\quad \text { h.c. } \\
& H_{C P}^{(1)}=\cos \theta_{c} \frac{G}{\sqrt{2}}\left[\bar{\nu} \gamma_{\alpha}\left(g_{V}^{\prime}-g_{A}^{\prime} \gamma_{5}\right) \tau\right] \quad\left[\bar{d} \gamma^{\alpha}\left(\chi_{V}^{d}-\chi_{A}^{d} \gamma_{5}\right) u\right]+\text { h.c. } \\
& H_{C P}^{(0)}=\cos \theta_{c} \frac{G}{\sqrt{2}}\left[\bar{\nu}\left(g_{S}+g_{P} \gamma_{5}\right) \tau\right] \quad\left[\bar{d}\left(\eta_{S}^{d}+\eta_{P}^{d} \gamma_{5}\right) u\right]+\quad \text { h.c. }
\end{aligned}
$$

plus a similar term with the complex parameters $\eta^{d}, \chi^{d}$ replaced by $\eta^{s}, \chi^{s}$ for the $\Delta S=1$ contribution.

The dominance of $(V-A)$ contributions to the leptonic current in leptonic and semileptonic $\tau$-decays has been demonstrated experimentally under fairly mild theoretical assumptions. A pure $(V-A)$ structure of the leptonic current in $H_{S M}$ will therefore be adopted for simplicity. A slight deviation from $(V-A)$ for the hadronic current can in principle 
be masked by the form factors. However, tight restrictions can be derived from the ratio $\Gamma(\tau \rightarrow \nu \pi) / \Gamma(\tau \rightarrow \nu \pi \pi)$ using as input $f_{\pi}$ and the pion form factor from $e^{+} e^{-}$annihilation'. The study of CP violation will entirely rely on interference terms between $H_{S M}$ and $H_{C P}^{(1)}, H_{C P}^{(0)}$. Interference terms between the dominant $(V-A)$ leptonic current in $H_{S M}$ and a possible $V+A$ term in the leptonic current of $H_{C P}^{(0,1)}$ are suppressed with the ratio between the mass of the $\tau$ neutrino and $m_{\tau}$. Therefore only contributions from left-handed neutrinos will be included, i.e. $g_{V}=g_{A}=g_{V}^{\prime}=g_{A}^{\prime}=g_{S}=-g_{P}=1$.

The hadronic matrix elements of the currents $\bar{d} \gamma^{\alpha} u$ (and similarly $\bar{d} \gamma^{\alpha} \gamma_{5} u$ ) in $H_{S M}$ and $H_{C P}^{(1)}$ are of course identical. The spin zero part is closely related to the corresponding matrix element of the scalar current in $H_{C P}^{(0)}$ through the equation of motion:

$$
Q^{\alpha} \bar{d} \gamma_{\alpha} u=\left(m_{u}-m_{d}\right) \bar{d} u ; \quad-Q^{\alpha} \bar{d} \gamma_{\alpha} \gamma_{5} u=\left(m_{u}+m_{d}\right) \bar{d} \gamma_{5} u
$$

with $Q^{\alpha}=i \partial^{\alpha}$. The Hamiltonian in Eq. (11) can thus be written in the form

$$
\begin{aligned}
H=\cos \theta_{c} \frac{G}{\sqrt{2}}\left[\bar{\nu} \gamma_{\alpha}\left(1-\gamma_{5}\right) \tau\right] & \left\{\left(\left(1+\chi_{V}^{d}\right) g^{\alpha \beta}+\frac{Q^{\alpha} Q^{\beta}}{m_{\tau}\left(m_{u}-m_{d}\right)} \eta_{S}^{d}\right) \bar{d} \gamma_{\beta} u\right. \\
- & \left.\left(\left(1+\chi_{A}^{d}\right) g^{\alpha \beta}+\frac{Q^{\alpha} Q^{\beta}}{m_{\tau}\left(m_{u}+m_{d}\right)} \eta_{P}^{d}\right) \bar{d} \gamma_{\beta} \gamma_{5} u\right\}+\quad \text { h.c. }
\end{aligned}
$$

and similarly for the Cabibbo suppressed mode. From this form it is evident that $\chi$ and $\eta$ play a fairly different role. Effects from $\chi_{V}$ and/or $\chi_{A}$ can only arise if both vector and axial hadronic currents contribute to the same final state and hence only for final states which are not eigenstates of $G$ parity and involve three or more mesons. Conversely, for all two meson decays and, adopting isospin symmetry, even all multipion states, $\mathrm{CP}$ violation cannot 2 arise

\footnotetext{
${ }^{1}$ The relative sign between hadronic vector and axial vector current is not fixed through this consideration. It can be determined by interference measurements in the $K \pi \pi$ and $K K \pi$ channels, e.g. of the structure functions $W_{F, G, H, I}[9]$.

${ }^{2}$ In this aspect we disagree with Ref. [6] where it has been claimed that CP violation in the $2 \pi$ channel can be induced through the exchange of an exotic intermediate vector boson.
} 
from a complex $\chi$. In contrast, $\mathrm{CP}$ violation can arise from a complex $\eta$, since $J=0$ and $J=1$ partial waves are affected differently in this case. For this reason contributions from nonvanishing $\chi$ will be ignored in the following.

\section{TWO MESON DECAYS: KINEMATICS, FORM FACTORS AND STRUCTURE FUNCTIONS}

Transitions from the vacuum to two pseudoscalar mesons $h_{1}$ and $h_{2}$ are induced through vector and scalar currents only, where the latter can be related to the former with the help of Eq. (2). Expanding this hadronic matrix element along the set of independent momenta $\left(q_{1}-q_{2}\right)_{\beta}$ and $Q_{\beta}=\left(q_{1}+q_{2}\right)_{\beta}$

$$
J_{\beta}=\left\langle h_{1}\left(q_{1}\right) h_{2}\left(q_{2}\right)\left|\bar{u} \gamma_{\beta} d\right| 0\right\rangle=\left(q_{1}-q_{2}\right)^{\delta} T_{\delta \beta} F\left(Q^{2}\right)+Q_{\beta} F_{S}\left(Q^{2}\right)
$$

the general amplitude for the strangeness conserving decay

$$
\tau^{-}(l, s) \rightarrow \nu\left(l^{\prime}, s^{\prime}\right)+h_{1}\left(q_{1}, m_{1}\right)+h_{2}\left(q_{2}, m_{2}\right)
$$

can be written as"

$$
\begin{aligned}
\mathcal{M} & =\cos \theta_{c} \frac{G}{\sqrt{2}} \bar{u}\left(l^{\prime}, s^{\prime}\right) \gamma_{\alpha}\left(1-\gamma_{5}\right) u(l, s)\left(g^{\alpha \beta}+\frac{Q^{\alpha} Q^{\beta}}{m_{\tau}\left(m_{u}-m_{d}\right)} \eta_{S}\right)\left[\left(q_{1}-q_{2}\right)^{\delta} T_{\delta \beta} F+Q_{\beta} F_{S}\right] \\
& =\cos \theta_{c} \frac{G}{\sqrt{2}} \bar{u}\left(l^{\prime}, s^{\prime}\right) \gamma_{\alpha}\left(1-\gamma_{5}\right) u(l, s)\left[\left(q_{1}-q_{2}\right)_{\beta} T^{\alpha \beta} F+Q^{\alpha} \tilde{F}_{S}\right]
\end{aligned}
$$

with

$$
\tilde{F}_{S}=\left(1+\frac{Q^{2}}{m_{\tau}\left(m_{u}-m_{d}\right)} \eta_{S}\right) F_{S}
$$

and similarly for the $\Delta S=1$ part. In Eq. (6) $s$ denotes the polarization 4-vector of the $\tau$ lepton satisfying $l_{\mu} s^{\mu}=0$ and $s_{\mu} s^{\mu}=-P^{2} . P$ denotes the polarization of the $\tau$ in the $\tau$ rest

\footnotetext{
${ }^{3}$ We suppress the superscript $d$ (or $s$ ) in $\eta_{S}$ in the following.
} 
frame with respect to its direction of flight in the laboratory frame and $T^{\alpha \beta}$ is the projector onto the spin one part

$$
T^{\alpha \beta}=g^{\alpha \beta}-\frac{Q^{\alpha} Q^{\beta}}{Q^{2}}
$$

As stated before, terms proportioal to $\chi$ do not contribute to $\mathrm{CP}$ violation in the two meson case and have therefore been neglegted.

The representation of the hadronic amplitude $\left\langle h_{1} h_{2}\left|\bar{u} \gamma_{\beta} d\right| 0\right\rangle=\left(q_{1}-q_{2}\right)^{\delta} T_{\delta \beta} F+Q_{\beta} \tilde{F}_{S}$ corresponds to a decomposition into spin one and spin zero contributions, e.g. the vector form factor $F\left(Q^{2}\right)$ corresponds to the $J^{P}=1^{-}$component of the weak charged current, and the scalar form factor $F_{S}\left(Q^{2}\right)$ to the $J^{P}=0^{+}$component. Up to the small isospin breaking terms, induced for example by the small quark mass difference, CVC implies the vanishing of $F_{S}$ for the two pion case. The small $u$ and $d$ quark masses enter presumably the couplings from charged Higgs exchange and thus cancel the apparent enhancement by the inverse power of $\left(m_{u}-m_{d}\right)$ in Eqs. (3,6, (7). The perspectives are more promising for the $\Delta S=1$ transition $\tau \rightarrow K \pi \nu$. The $J=1$ form factor $F$ is dominated by the $K^{*}(892)$ vector resonance contribution. However, in this case the scalar form factor $F_{S}$ is expected to receive a sizable resonance contribution $\left(\sim 5 \%\right.$ to the decay rate) from the $K_{0}^{*}(1430)$ with $J^{P}=0^{+}[11]$. In the subsequent discussion we will include both $\pi \pi$ and $K \pi$ final states. The corresponding $\tau^{+}$decay is obtained from Eq. (6) through the substitutions

$$
\left(1-\gamma_{5}\right) \rightarrow\left(1+\gamma_{5}\right), \quad \eta_{S} \rightarrow \eta_{S}^{*}
$$

Reaction (5) is most easily analyzed in the hadronic rest frame $\vec{q}_{1}+\vec{q}_{2}=0$. After integration over the unobserved neutrino direction, the differential decay rate in the rest frame of $h_{1}+h_{2}$ is given by 9,11

$$
\begin{aligned}
& d \Gamma\left(\tau^{-} \rightarrow 2 h \nu_{\tau}\right)=\left\{\bar{L}_{B} W_{B}+\bar{L}_{S A} W_{S A}+\bar{L}_{S F} W_{S F}+\bar{L}_{S G} W_{S G}\right\} \\
& \frac{G^{2}}{2 m_{\tau}}\left(\begin{array}{c}
\cos ^{2} \theta_{c} \\
\sin ^{2} \theta_{c}
\end{array}\right) \frac{1}{(4 \pi)^{3}} \frac{\left(m_{\tau}^{2}-Q^{2}\right)^{2}}{m_{\tau}^{2}}\left|\vec{q}_{1}\right| \frac{d Q^{2}}{\sqrt{Q^{2}}} \frac{d \cos \theta}{2} \frac{d \alpha}{2 \pi} \frac{d \cos \beta}{2} .
\end{aligned}
$$

The coefficients $\bar{L}_{X}$ contain all $\alpha, \beta$ and $\theta$ angular and $\tau$-polarization dependence and will be specified below. The hadronic structure functions $W_{X}, X \in\{B, S A, S F, S G\}$, depend 
only on $Q^{2}$ and the form factors $F$ and $\tilde{F}_{S}$ of the hadronic current. The dependence can be obtained from Eq. (34) in [9] with the replacements $x_{4} \rightarrow 2 \vec{q}_{1}, F_{3} \rightarrow-i F, F_{4} \rightarrow \tilde{F}_{S}$. One has:

$$
\begin{aligned}
W_{B}\left[\tau^{-}\right] & =4\left(\vec{q}_{1}\right)^{2}|F|^{2} \\
W_{S A}\left[\tau^{-}\right] & =Q^{2}\left|\tilde{F}_{S}\right|^{2} \\
W_{S F}\left[\tau^{-}\right] & =4 \sqrt{Q^{2}}\left|\vec{q}_{1}\right| \operatorname{Re}\left[F \tilde{F}_{S}^{*}\right] \\
W_{S G}\left[\tau^{-}\right] & =-4 \sqrt{Q^{2}}\left|\vec{q}_{1}\right| \operatorname{Im}\left[F \tilde{F}_{S}^{*}\right]
\end{aligned}
$$

where $\left|\vec{q}_{1}\right|=q_{1}^{z}$ is the momentum of $h_{1}$ in the rest frame of the hadronic system:

$$
\vec{q}_{1}^{z}=\frac{1}{2 \sqrt{Q^{2}}}\left(\left[Q^{2}-m_{1}^{2}-m_{2}^{2}\right]^{2}-4 m_{1}^{2} m_{2}^{2}\right)^{1 / 2} .
$$

The hadronic structure functions $W_{X}\left[\tau^{+}\right]$are obtained by the replacement $\eta_{S} \rightarrow \eta_{S}^{*}$ in $\tilde{F}_{S}$ in Eqs. (12-14, (7). CP conservation implies that all four structure functions are identical for $\tau^{+}$and $\tau^{-}$. With the ansatz for the form factors formulated in Eq. (6) CP violation can be present in $W_{S F}$ and $W_{S G}$ only and requires complex $\eta_{S}$. As will be shown in the subsequent discussion $\mathrm{CP}$ violation in $W_{S G}$ in maximal for fixed $\eta_{S}$ in the absence of hadronic phases whereas $W_{S F}$ in contrast requires complex $\eta_{S}$ and hadronic phases simultaneously.

We will now demonstrate that $W_{S F}$ can be measured in $e^{+} e^{-}$annihilation experiments in the study of single unpolarized $\tau$ decays even if the $\tau$ rest frame cannot be reconstructed. In this respect the result differ from earlier studies of the two meson modes where either polarized beams and reconstruction of the full kinematics [6] or correlated fully reconstructed $\tau^{-}$and $\tau^{+}$decays were required [5]. The determination of $W_{S G}$, however, requires the knowledge of the full $\tau$ kinematics and $\tau$ polarization.

For the definition and discussion of the angles and leptonic coefficients $\bar{L}_{X}$ in Eq. (10) we therefore consider two different situations, depending on whether the direction of flight of the $\tau$ in the hadronic rest frame [denoted by $\vec{n}_{\tau}$ ] can be measured or not. 


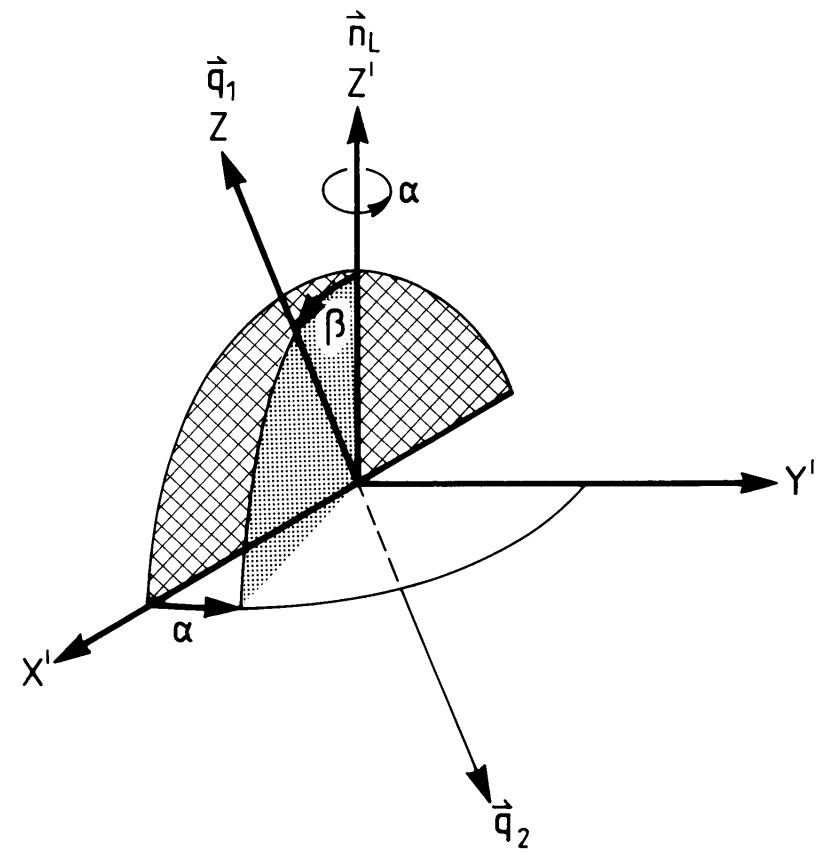

FIG. 1. Definition of the angles $\alpha$ and $\beta$ in two meson decays.

I) The $\tau$ direction in the hadronic rest frame is not known:

In this case, the angle $\beta$ in Eq. (10) denotes the angle between the direction of $h_{1}\left(\hat{q}_{1}=\right.$ $\vec{q}_{1} /\left|\vec{q}_{1}\right|$ ) and the direction of the laboratory $\vec{n}_{L}$ viewed from the hadronic rest frame (see Fig. 1)

$$
\cos \beta=\vec{n}_{L} \cdot \hat{q}_{1}
$$

$\vec{n}_{L}$ is obtained from $\vec{n}_{L}=-\vec{n}_{Q}$, where $\vec{n}_{Q}$ denotes the direction of the two-meson-system in the laboratory. The azimuthal angle $\alpha$ is not observable and has to be averaged out.

The angle $\theta(0 \leq \theta \leq \pi)$ in Eq. (10) which is only relevant for the analysis of polarized $\tau$ 's, is the angle between the direction of flight of the $\tau$ in the laboratory frame and the direction of the hadrons as seen in the $\tau$ rest frame. The cosine of the angle $\theta$ is observable even in experiments, where the $\tau$ direction cannot be measured experimentally. This is because $\cos \theta$ can be calculated [13, 12,9] from the energy $E_{h}$ of the hadronic system in the laboratory frame 


$$
\cos \theta=\frac{\left(2 x m_{\tau}^{2}-m_{\tau}^{2}-Q^{2}\right)}{\left(m_{\tau}^{2}-Q^{2}\right) \sqrt{1-4 m_{\tau}^{2} / s}}, \quad x=2 \frac{E_{h}}{\sqrt{s}}, \quad s=4 E_{\text {beam }}^{2} .
$$

Of particular importance for the subsequent discussion is $\psi$, the angle between the direction of the laboratory and the $\tau$ as seen from the hadronic rest frame. Again the cosine of this angle can be calculated from the hadronic energy $E_{h}$ [13, 12,9]. One has:

$$
\cos \psi=\frac{x\left(m_{\tau}^{2}+Q^{2}\right)-2 Q^{2}}{\left(m_{\tau}^{2}-Q^{2}\right) \sqrt{x^{2}-4 Q^{2} / s}}
$$

The angular coefficients $\bar{L}_{B, S A, S F, S G}$ for $\tau^{-}$decay in Eq. (10) are given by [9]:

$$
\begin{aligned}
\bar{L}_{B} & =2 / 3 K_{1}+K_{2}-2 / 3 \bar{K}_{1}\left(3 \cos ^{2} \beta-1\right) / 2 \\
\bar{L}_{S A} & =K_{2} \\
\bar{L}_{S F} & =-\bar{K}_{2} \cos \beta \\
\bar{L}_{S G} & =0
\end{aligned}
$$

with

$$
\begin{aligned}
& K_{1}=1-\gamma_{V A} P \cos \theta-\left(m_{\tau}^{2} / Q^{2}\right)\left(1+\gamma_{V A} P \cos \theta\right) \\
& K_{2}=\left(m_{\tau}^{2} / Q^{2}\right)\left(1+\gamma_{V A} P \cos \theta\right) \\
& \bar{K}_{1}=K_{1}\left(3 \cos ^{2} \psi-1\right) / 2-3 / 2 K_{4} \sin 2 \psi \\
& \bar{K}_{2}=K_{2} \cos \psi+K_{4} \sin \psi \\
& K_{4}=\sqrt{m_{\tau}^{2} / Q^{2}} \gamma_{V A} P \sin \theta \\
& K_{5}=\sqrt{m_{\tau}^{2} / Q^{2}} P \sin \theta
\end{aligned}
$$

where

$$
\gamma_{V A}=\frac{2 g_{V} g_{A}}{g_{V}^{2}+g_{A}^{2}}
$$

( $K_{5}$ in Eq. (20) is needed in the later discussion). In our case of purely left-handed leptonic currents $\gamma_{V A}=1$. For $P=0$, the case relevant in the low energy region $\sqrt{S} \approx 10 \mathrm{GeV}$, Eq. (19) simplifies to 


$$
\begin{aligned}
\bar{L}_{B} & =\frac{1}{3}\left(2+\frac{m_{\tau}^{2}}{Q^{2}}\right)-\frac{1}{6}\left(1-\frac{m_{\tau}^{2}}{Q^{2}}\right)\left(3 \cos ^{2} \psi-1\right)\left(3 \cos ^{2} \beta-1\right) \\
\bar{L}_{S A} & =\frac{m_{\tau}^{2}}{Q^{2}} \\
\bar{L}_{S F} & =-\frac{m_{\tau}^{2}}{Q^{2}} \cos \psi \cos \beta .
\end{aligned}
$$

Note that the angular coefficient $\bar{L}_{S G}$ vanishes, if the hadronic rest frame is experimentally not known and only the distribution in $\beta$ is considered.

The differential rate (Eq. (10)) for the CP conjugated process can be obtained from the previous results by reversing all momenta $\vec{p} \rightarrow-\vec{p}$, the $\tau$ spin vector $\vec{s} \rightarrow-\vec{s}$, the polarization $P \rightarrow-P$, and the transition $\gamma_{V A} \rightarrow-\gamma_{V A}$. CP therefore relates the differential decay rates for $\tau^{+}$and $\tau^{-}$as follows:

$$
d \Gamma\left[\tau^{-}\right]\left(\gamma_{V A}, P, W_{X}\left[\tau^{-}\right]\right) \rightarrow d \Gamma\left[\tau^{+}\right]\left(-\gamma_{V A},-P, W_{X}\left[\tau^{+}\right]\right)
$$

Note that the coefficients $\bar{L}_{X}$ contain the full $\gamma_{V A}$ and $P$ dependence. From the interference between the spin-zero spin-one terms (denoted by the subscript $X=S F, S G$ ) one can construct the following CP-violating quantities:

$$
\begin{aligned}
\Delta_{X} & =\frac{1}{2}\left(\bar{L}_{X}\left(\gamma_{V A}, P\right) W_{X}\left[\tau^{-}\right]-\bar{L}_{X}\left(-\gamma_{V A},-P\right) W_{X}\left[\tau^{+}\right]\right) \\
& =\bar{L}_{X}\left(\gamma_{V A}, P\right) \frac{1}{2}\left(W_{X}\left[\tau^{-}\right]-W_{X}\left[\tau^{+}\right]\right) \\
& \equiv \bar{L}_{X} \Delta W_{X}
\end{aligned}
$$

As mentioned before the hadronic structure functions $W_{X}\left[\tau^{-}\right]$and $W_{X}\left[\tau^{+}\right]$differ only in the complex parameter $\eta_{S}$ in

$$
\tilde{F}_{S}\left[\tau^{-}\right]=\left(1+\frac{Q^{2}}{m_{\tau}\left(m_{u}-m_{d}\right)} \eta_{S}\right) F_{S} \quad \text { and } \quad \tilde{F}_{S}\left[\tau^{+}\right]=\left(1+\frac{Q^{2}}{m_{\tau}\left(m_{u}-m_{d}\right)} \eta_{S}^{*}\right) F_{S}
$$

and one obtains for the only nonvanishing spin-zero spin-one term $L_{S F} W_{S F}$

$$
\Delta W_{S F}=4 \sqrt{Q^{2}}\left|\vec{q}_{1}\right| \frac{Q^{2}}{m_{\tau}\left(m_{u}-m_{d}\right)} \operatorname{Im}\left(F F_{S}^{*}\right) \operatorname{Im}\left(\eta_{S}\right)
$$


In essence this measurement analyses the difference in the correlated energy distribution of the mesons $h_{1}$ and $h_{2}$ from $\tau^{+}$and $\tau^{-}$decay in the laboratory. As already mentioned, $\Delta W_{S F}$ is observable for single $\tau^{+}$and $\tau^{-}$decays without knowledge of the $\tau$ rest frame. Any nonvanishing experimental result for $\Delta W_{S F}$ would be a clear signal of CP violation. Note that a nonvanishing $\Delta W_{S F}$ requires nontrivial hadronic phases (in addition to the CP violating phases $\left.\eta_{S}\right)$ in the form factors $F$ and $F_{S}$. Such hadronic phases in $F\left(F_{S}\right)$ originate in the $K \pi \nu_{\tau}$ decay mode from complex Breit Wigner propagators for the $K^{*}\left(K_{0}^{*}\right)$ resonance. Sizable effects of these hadronic phases are expected in this decay mode [11].

Once the $\tau$ rest frame is known and a preferred direction of polarization exists one may proceed further, determine also $W_{S G}$ and thus perform a second independent test for CP violation. The subsequent analysis is performed for a $\tau$ with spin direction $\vec{s}$.

II) The $\tau$ direction in the hadronic rest frame is known:

As discussed in the appendix, the $\tau$ direction can be determined in a large number of cases with the help of vertex detectors. In this case, the vector $\vec{n}_{L}$ is replaced by $\vec{n}_{\tau}$ and hence $\psi \Rightarrow 0$, whereas $\cos \theta=-\vec{s} \vec{n}_{\tau}$. The angles $\alpha$ and $\beta$ are in this case defined by (see Fig. 1 with the replacement $\vec{n}_{L} \rightarrow \vec{n}_{\tau}$; the $X^{\prime}-Z^{\prime}$-plane is defined by the spin vector $\vec{s}$ and $\vec{n}_{\tau}$ ):

$$
\begin{aligned}
& \cos \beta=\vec{n}_{\tau} \cdot \hat{q}_{1}, \\
& \cos \alpha=\frac{\left(\vec{n}_{\tau} \times \vec{s}\right) \cdot\left(\vec{n}_{\tau} \times \hat{q}_{1}\right)}{\left|\vec{n}_{\tau} \times \vec{s}\right|\left|\vec{n}_{\tau} \times \hat{q}_{1}\right|}, \\
& \sin \alpha=-\frac{\vec{s} \cdot\left(\vec{n}_{\tau} \times \hat{q}_{1}\right)}{\left|\vec{n}_{\tau} \times \vec{s}\right|\left|\vec{n}_{\tau} \times \hat{q}_{1}\right|},
\end{aligned}
$$

and the coefficients $\bar{L}_{X}$ for the $\tau^{-}$decay are given by:

$$
\begin{aligned}
& \bar{L}_{B}=K_{1} \sin ^{2} \beta+K_{2}-K_{4} \sin 2 \beta \cos \alpha \\
& \bar{L}_{S A}=K_{2} \\
& \bar{L}_{S F}=-K_{2} \cos \beta-K_{4} \sin \beta \cos \alpha \\
& \bar{L}_{S G}=K_{5} \sin \beta \sin \alpha
\end{aligned}
$$

The coefficients for $K_{1}, K_{2}, K_{3}, K_{4}, K_{5}$ for $\psi \neq 0$ are given in (20). For $P=0$, the case relevant in the low energy region $\sqrt{S} \approx 10 \mathrm{GeV}$, Eq. (32) simplifies to 


$$
\begin{aligned}
\bar{L}_{B} & =\left(1-\frac{m_{\tau}^{2}}{Q^{2}}\right) \sin ^{2} \beta+\frac{m_{\tau}^{2}}{Q^{2}} \\
\bar{L}_{S A} & =\frac{m_{\tau}^{2}}{Q^{2}} \\
\bar{L}_{S F} & =-\frac{m_{\tau}^{2}}{Q^{2}} \cos \beta \\
\bar{L}_{S G} & =0
\end{aligned}
$$

Evidently $W_{S G}$ cannot be measured in this case and thus no additional test of CP violation is possible. However, longitudinally polarized incident beams [6] or the study of $\tau^{+}$and $\tau^{-}$correlations [5] could allow to recover polarization. The distributions in [6] are in fact equivalent to the product of $\bar{L}_{S G} W_{S G}$ if the angle $\alpha$ is defined with respect to the $\tau$ spin vector through Eqs. (30,31). Correlations between the $\tau^{+}$and $\tau^{-}$decay products may even allow to define the angle $\alpha$ without $\tau$ polarization.

The differential rate (Eq. (10)) for the $\mathrm{CP}$ conjugated process can be obtained from the previous results by reversing all momenta $\vec{p} \rightarrow-\vec{p}$, the $\tau$ spin vector $\vec{s} \rightarrow-\vec{s}$, the polarization $P \rightarrow-P$, $\sin \alpha \rightarrow-\sin \alpha$ and the transition $\gamma_{V A} \rightarrow-\gamma_{V A}$. CP therefore relates the differential decay rates for $\tau^{+}$and $\tau^{-}$as:

$$
d \Gamma\left[\tau^{-}\right]\left(\sin \alpha, \gamma_{V A}, P, W_{X}\left[\tau^{-}\right]\right) \rightarrow d \Gamma\left[\tau^{+}\right]\left(-\sin \alpha,-\gamma_{V A},-P, W_{X}\left[\tau^{+}\right]\right)
$$

From the interference between the spin-zero spin-one terms one can construct the following CP-violating quantities:

$$
\begin{aligned}
& \Delta_{X}= \frac{1}{2}\left(\bar{L}_{X}\left(\sin \alpha, \gamma_{V A}, P\right) W_{X}\left[\tau^{-}\right]-\bar{L}_{X}\left(-\sin \alpha,-\gamma_{V A},-P\right) W_{X}\left[\tau^{+}\right]\right) \\
&= \bar{L}_{X}\left(\sin \alpha, \gamma_{V A}, P\right) \frac{1}{2}\left(W_{X}\left[\tau^{-}\right]-W_{X}\left[\tau^{+}\right]\right) \\
& \equiv \bar{L}_{X} \Delta W_{X} \\
& \Delta W_{S F}=4 \sqrt{Q^{2}}\left|\vec{q}_{1}\right| \frac{Q^{2}}{m_{\tau}\left(m_{u}-m_{d}\right)} \operatorname{Im}\left(F F_{S}^{*}\right) \operatorname{Im}\left(\eta_{S}\right) \\
& \Delta W_{S G}=4 \sqrt{Q^{2}}\left|\vec{q}_{1}\right| \frac{Q^{2}}{m_{\tau}\left(m_{u}-m_{d}\right)} \operatorname{Re}\left(F F_{S}^{*}\right) \operatorname{Im}\left(\eta_{S}\right) .
\end{aligned}
$$


Any observed nonzero value of these quantities would signal a true CP violation. Eqs.(41) and (42) show that the sensitivity to $\mathrm{CP}$ violating effects in $\Delta W_{S F}$ and $\Delta W_{S G}$ can be fairly different depending on the hadronic phases. Whereas $\Delta W_{S F}$ requires nontrivial hadronic phases $\Delta W_{S G}$ is maximal for fixed $\eta_{S}$ in the absence of hadronic phases.

\section{THREE MESON DECAYS}

The structure function formalism [9] allow also for a systematic analysis of possible CP violation effects in the three meson case. Some of these effects have already been briefly discussed in [14]. The $K \pi \pi$ and $K K \pi$ decay modes with nonvanishing vector and axial vector current are of particular importance for the detection of possible $\mathrm{CP}$ violation originating from exotic intermediate vector bosons. This would be signalled by a nonvanishing difference between the structure functions $W_{X}\left(\tau^{-}\right)$and $W_{X}\left(\tau^{+}\right)$with $X \in\{F, G, H, I\}$. A difference in the structure functions with $X \in\{S B, S C, S D, S E, S F, S G\}$ can again be induced through a CP violating scalar exchange. More details will be presented in a subsequent publication.

\section{APPENDIX A: TAU KINEMATICS IN ONE PRONG DECAYS FROM IMPACT PARAMETERS}

The reconstruction of the full kinematics is evidently a significant advantage in the analysis of $\tau$ decays. It has been shown in 15$]$ that this is possible for the $\tau^{+}\left(\rightarrow \pi^{+} \bar{\nu}\right) \tau^{-}\left(\rightarrow \pi^{-} \nu\right)$ final state if the tracks of $\pi^{+}$and $\pi^{-}$are experimentally measured - even if the production vertex is not known. If the production vertex is known, the knowledge of one track of $\pi^{+}$ or $\pi^{-}$is sufficient. Also three prong decays with measured tracks allow the full kinematic reconstruction. In this appendix the discussion of [15] is generalized to the case, where one $\tau$ decays into one charged hadron and the second $\tau$ decays into $\rho$ or $K^{*}$ with the subsequent decay into a neutral and a charged meson. For definiteness assume the $\tau^{-}$decays into $\pi^{-} \nu$ and the $\tau^{+}$decays into $\rho^{+}\left(\rightarrow \pi^{+} \pi^{0}\right) \bar{\nu}$. This is a generalization of the situation discussed in [15], where both $\tau$ 's were assumed to decay into one charged hadron each. In fact, the 
method is also applicable in the multibody one prong decay, if the momenta of all neutral decays are determined experimentally.

Assume that the momenta of all hadrons are measured and, furthermore, the tracks of $\pi^{+}$and $\pi^{-}$are also measured. We shall demonstrate that also in this case the impact vector allows to reconstruct the original direction of $\tau^{+}$and $\tau^{-}$in most of the cases.

We use the following notation: $\vec{n}_{+}$and $\vec{n}_{-}$denote unit vectors into the directions of the $\rho^{+}$and the $\pi^{-}$momentum. $\vec{r}$ denotes the direction of the $\pi^{+}$track. The cosines of the angles $\theta_{ \pm}^{L}$ between the $\tau^{ \pm}$directions and the hadronic systems in the lab frame are denoted by $c_{ \pm} \equiv \cos \theta_{ \pm}^{L}=\vec{n}_{\tau_{ \pm}} \vec{n}_{ \pm} . \quad c_{+}$and $c_{-}$can be calculated from the energy and mass of the hadronic system as given in Eq. (1) of [15].

In a first step, we determine the vector $\vec{d}$ which connects the $\tau_{+}$and $\tau_{-}$decay vertex. As shown in 15] $\vec{d}$ can be geometrically be reconstructed from $c_{+}, c_{-}, \vec{n}_{+}, \vec{n}_{-}$up to a two-fold ambiguity. Only the directions, but not the length of $\vec{d}$ can be obtained in this way, therefore we define $\vec{D}=\vec{d} /|\vec{d}|$. From Eq. (9) in [15] we find the unit vector

$$
\begin{aligned}
\vec{D} & =\vec{D}_{\min }+\frac{1}{1-\left(\vec{n}_{+} \vec{n}_{-}\right)^{2}}\left[-\left(c_{+}\left(\vec{n}_{+} \vec{n}_{-}\right)+c_{-}\right) \vec{n}_{-}+\left(c_{-}\left(\vec{n}_{+} \vec{n}_{-}\right)+c_{+}\right) \vec{n}_{+}\right] \\
& \equiv \vec{D}_{\min }+\vec{D}_{0}
\end{aligned}
$$

$\vec{D}_{\text {min }}$ denotes the direction of the vector which connects the points of closest approach between the straight lines through the decay vertex of $\tau^{+}$and $\tau^{-}$directions $\vec{n}_{+}$and $\vec{n}_{-}$, respectively and hence is normal to $\vec{n}_{+}$and $\vec{n}_{-} . \vec{D}_{0}$ is the remaining piece of $\vec{D}$ which by construction lies in the plane spanned by $\vec{n}_{+}$and $\vec{n}_{-}$.

Note that $\vec{D}_{\min } \perp \vec{D}_{0}$ by construction and hence

$$
\vec{D}_{\text {min }}= \pm \frac{\left(\vec{n}_{+} \times \vec{n}_{-}\right)}{\left|\vec{n}_{+} \times \vec{n}_{-}\right|}\left|\vec{D}_{\text {min }}\right|
$$

From Eq. (A2) and $\vec{D}^{2}=1, \vec{D}_{\text {min }} \cdot \vec{D}_{0}=0$ one has

$$
\begin{aligned}
\vec{D}_{\text {min }}^{2} & =1-\vec{D}_{0}^{2} \\
& =\frac{1}{1-\left(\vec{n}_{+} \vec{n}_{-}\right)^{2}}\left\{1-\left[c_{+}^{2}+c_{-}^{2}+\left(\vec{n}_{+} \vec{n}_{-}\right)^{2}+2 c_{+} c_{-}\left(\vec{n}_{+} \vec{n}_{-}\right)\right]\right\}
\end{aligned}
$$


With $\left|\vec{n}_{+} \times \vec{n}_{-}\right|=\sqrt{1-\left(\vec{n}_{+} \vec{n}_{-}\right)^{2}}$ on obtains

$$
\begin{aligned}
\vec{D}= & \pm \frac{\left(\vec{n}_{+} \times \vec{n}_{-}\right)}{\left(1-\left(\vec{n}_{+} \vec{n}_{-}\right)^{2}\right)} \sqrt{1-\left[c_{+}^{2}+c_{-}^{2}+\left(\vec{n}_{+} \vec{n}_{-}\right)^{2}+2 c_{+} c_{-}\left(\vec{n}_{+} \vec{n}_{-}\right)\right]} \\
& -\frac{1}{\left(1-\left(\vec{n}_{+} \vec{n}_{-}\right)^{2}\right)}\left[\left(c_{+}\left(\vec{n}_{+} \vec{n}_{-}\right)+c_{-}\right) \vec{n}_{-}-\left(c_{-}\left(\vec{n}_{+} \vec{n}_{-}\right)+c_{+}\right) \vec{n}_{+}\right] \\
= & \pm \vec{D}_{\perp}+\vec{D}_{0}
\end{aligned}
$$

No information on the tracks has been used. In a second step one predicts from measured momenta the vector $\vec{v}$ of minimal distance between the positive and negative track with the help of $\vec{r}, \vec{n}_{-}$, and $\vec{D}$. Depending on the two choices for $\vec{D}$ one will obtain two predictions for the direction of $\vec{v}$. Frequently, only one of them will be compatible with the actual measurement.

In order to calculate the vector $\vec{v}$ we choose a coordinate frame where the $\tau_{-}$decay point lies at the origin and the negative and positive tracks are parameterized as $\lambda \vec{n}_{-}$and $\vec{D}+\lambda \vec{r}$ respectively. [In reality one should scale $\vec{D}$ with $|\vec{d}|$, the actual decay length of $\tau^{+}$plus $\tau^{-}$. However, since we are only interested in directions, this factor drops out.] A straightforward geometrical calculation predicts

$$
\vec{v}=\vec{D}+\left[\left((\vec{D} \vec{r})\left(\vec{r} \vec{n}_{-}\right)-\left(\vec{D} \vec{n}_{-}\right)\right) \vec{n}_{-}+\left(\left(\vec{D} \vec{n}_{-}\right)(\vec{n}-\vec{r})-(\vec{D} \vec{r})\right) \vec{r}\right] \frac{1}{1-\left(\vec{r} \vec{n}_{-}\right)^{2}}
$$

Up to now no use has been made of track measurements. At this point $\vec{r}$ is given by the momentum of the $\pi^{+}$. Inserting the two solutions $\vec{D}_{ \pm}= \pm \vec{D}_{\perp}+\vec{D}_{0}$ one obtains two predictions for $\vec{v}$.

$$
\begin{gathered}
\vec{v}_{1}=\vec{D}_{+}+\left[\left(\left(\vec{D}_{+} \vec{r}\right)\left(\vec{r} \vec{n}_{-}\right)-\left(\vec{D}_{+} \vec{n}_{-}\right)\right) \vec{n}_{-}+\left(\left(\vec{D}_{+} \vec{n}_{-}\right)\left(\vec{n}_{-} \vec{r}\right)-\left(\vec{D}_{+} \vec{r}\right)\right) \vec{r}\right] \frac{1}{1-\left(\vec{r}_{-}\right)^{2}} \\
\vec{v}_{2}=\vec{D}_{-}+\left[\left(\left(\vec{D}_{-} \vec{r}\right)\left(\vec{r}_{-}\right)-\left(\vec{D}_{-} \vec{n}_{-}\right)\right) \vec{n}_{-}+\left(\left(\vec{D}_{-} \vec{n}_{-}\right)\left(\vec{n}_{-} \vec{r}\right)-\left(\vec{D}_{-} \vec{r}\right)\right) \vec{r}\right] \frac{1}{1-\left(\vec{r}_{-}\right)^{2}}(
\end{gathered}
$$

Note that both solutions $\vec{v}_{1}$ and $\vec{v}_{2}$ are normal to $\vec{n}_{-}$and parallel or antiparallel to each other, i.e. $\vec{v}_{1} \times \vec{v}_{2}=0$.

The next step is to compare the measured $\vec{v}$ with the calculated solutions $\vec{v}_{1}, \vec{v}_{2}$ and select the proper one. In order that this strategy can work we have to restrict ourselves to 


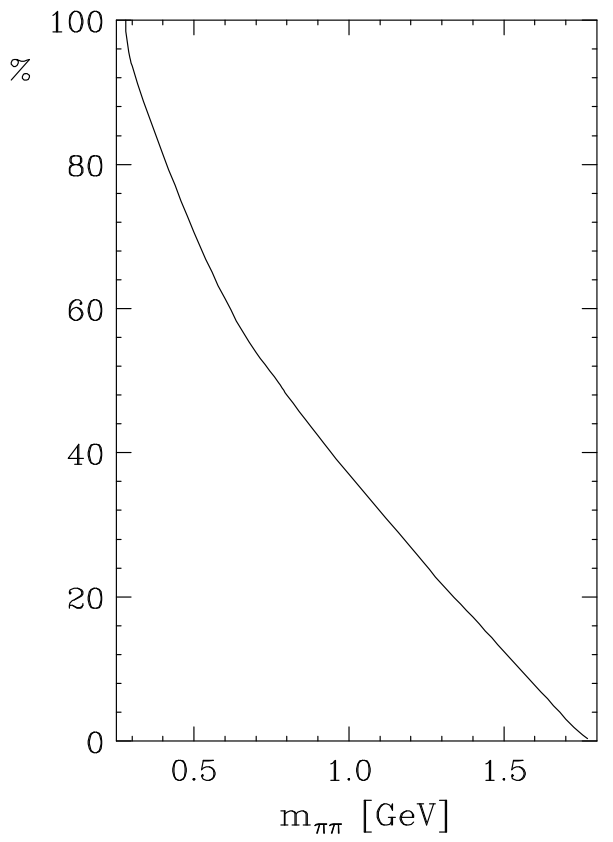

FIG. 2. Percentage of events where the difference in the direction of $\vec{v}_{1}$ and $\vec{v}_{2}$ allows the full reconstruction.

the case, where the two results for $\vec{v}$ in Eqs. A9, A10 correspond to two opposite directions for $\vec{v}$ (and not only to different lenghts which would not allow to resolve the ambiguity).

Using Monte Carlo methods we have checked for the $\pi^{-} \pi^{0}$ configuration that this is possible for $100 \%$ of the events with $\pi^{-} \pi^{0}$ at threshold, about $50 \%$ for events with $m_{\pi \pi} \sim m_{\rho}$ (the dominant configuration!) and zero at the kinematical endpoint $m_{\pi \pi}=m_{\tau}$, as illustrated in Fig. 2. On the other hand, in the limit $m_{\pi \pi} \rightarrow m_{\tau}$ the difference between the two solutions per se shrinks to zero, such that an approximate reconstruction seems possible in this case again.

\section{Acknowledgements}

The work of E. M. was supported in part by DFG Contract Ku 502/5-1. 


\section{REFERENCES}

[1] M. Kobayashi and T. Maskawa, Prog. Theor. Phys. 49, 652 (1973).

[2] W. Bernreuther and O. Nachtmann, Phys. Rev. Lett. 63, 2787 (1989); erratum: 64, 1072 (1990); W. Bernreuther, G.W. Boltz, O. Nachtmann and P. Overmann, Z. Phys. C52, 567 (1991);

C.A. Nelson, Phys. Lett. B267, 128 (1991);

S. Goozovat and C.A. Nelson, Phys. Rev. D44, 2818 (1991).

[3] N. Wermes, Proceedings of the TAU96 Workshop, Colorado, (1996), and references therein; OPAL Coll. Z. Phys. C66, 31 (1995).

[4] Y. Grossman, Nucl. Phys. B426, 355 (1994), and references therein.

[5] C.A. Nelson et al., Phys. Rev. D50, 4544 (1994), and references therein.

[6] Y.S. Tsai, Phys. Rev. D51, 3172 (1995).

[7] S.Y. Choi, K. Hagiwara and M. Tanabashi, Phys. Rev. D52, 1614 (1995).

[8] U. Kilian, J.G. Körner, K .Schilcher and Y.L. Wu, Z. Phys. C62, 413 (1994).

[9] J.H. Kühn and E. Mirkes, Z. Phys. C56, 661 (1992); erratum ibid. C67, 364 (1995).

[10] R. Decker, E. Mirkes, R. Sauer and Z. Was, Z. Phys. C58 445 (1993).

[11] M. Finkemeier and E. Mirkes, TTP95-44, hep-ph/9601275, to be published in Z. Phys. C.

[12] J.H. Kühn and E. Mirkes, Phys. Lett. B286, 281 (1992).

[13] J.H. Kühn and F. Wagner, Nucl. Phys. B236, 16 (1984).

[14] M. Finkemeier and E. Mirkes, Proceedings of the Workshop on the Tau/Charm Factory, Argonne, IL, (1995), hep-ph/9508312.

[15] J.H. Kühn, Phys. Lett. B313, 458 (1993). 